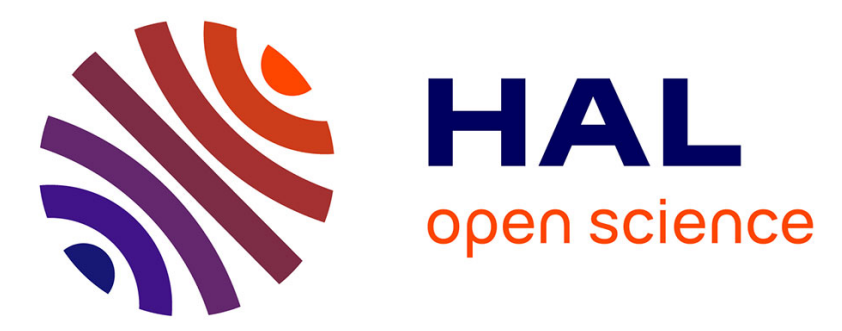

\title{
From Hopf-bifurcation to limit cycles control in underactuated mechanical systems
}

Nahla Khraief Haddad, Safya Belghith, Hassène Gritli, Ahmed Chemori

\section{To cite this version:}

Nahla Khraief Haddad, Safya Belghith, Hassène Gritli, Ahmed Chemori. From Hopf-bifurcation to limit cycles control in underactuated mechanical systems. International journal of bifurcation and chaos in applied sciences and engineering , 2017, 27 (07), 10.1142/S0218127417501048 . lirmm01723915

\section{HAL Id: lirmm-01723915 https://hal-lirmm.ccsd.cnrs.fr/lirmm-01723915}

Submitted on 5 Mar 2018

HAL is a multi-disciplinary open access archive for the deposit and dissemination of scientific research documents, whether they are published or not. The documents may come from teaching and research institutions in France or abroad, or from public or private research centers.
L'archive ouverte pluridisciplinaire HAL, est destinée au dépôt et à la diffusion de documents scientifiques de niveau recherche, publiés ou non, émanant des établissements d'enseignement et de recherche français ou étrangers, des laboratoires publics ou privés. 


\title{
From Hopf-bifurcation to limit cycles control in underactuated mechanical systems
}

\author{
Nahla Khraief Haddad ${ }^{*}$ \\ RISC Lab, National School of Engineers of Tunis. \\ University of Tunis El Manar, BP.3\%, le Belvédère, \\ 1002 Tunis, Tunisia \\ Safya Belghith \\ RISC Lab, National School of Engineers of Tunis. \\ University of Tunis El Manar, BP.37, le Belvédère, \\ 1002 Tunis, Tunisia \\ Hassène Gritli \\ RISC Lab, National School of Engineers of Tunis. \\ University of Tunis El Manar, BP.37, le Belvédère, \\ 1002 Tunis, Tunisia \\ Ahmed Chemori \\ LIRMM, University of Montpellier 2, CNRS, \\ 161 rue Ada, 34392 Montpellier, France \\ Received (to be inserted by publisher)
}

\begin{abstract}
This paper deals with the problem of obtaining stable and robust oscillations of underactuated mechanical systems. It is concerned with the Hopf bifurcation analysis of a Controlled Inertia Wheel Inverted Pendulum (C-IWIP). Firstly, the stabilization was achieved with a control law based on the Interconnection, Damping, Assignment Passive Based Control method (IDA-PBC). Interestingly, the considered closed loop system exhibits both supercritical and subcritical Hopf bifurcation for certain gains of the control law. Secondly, we used the center manifold theorem and the normal form technique to study the stability and instability of limit cycles emerging from the Hopf bifurcation. Finally, numerical simulations were conducted to validate the analytical results in order to prove that with IDA-PBC we can control not only the unstable equilibrium but also some trajectories such us limit cycles.
\end{abstract}

Keywords: Hopf-bifurcation, limit cycles, IDA-PBC control, normal form, center manifold.

\section{Introduction}

Different methodologies have been considered in the literature [Fradkov \& Pogromsky, 1998$]$ and [Leonard et al. 1998] to stabilize oscillations for underactuated mechanical system [Choukchou-Braham et al. [2014].

\footnotetext{
*khraiefnahla@gmail.com
} 
Such trajectories have a practical importance, including its applications in mechanical engineering, acous-

5 tics, electronics, and biomechanics (perhaps even in finance and macroeconomics). One application deals with the Inertia Wheel Inverted Pendulum (IWIP). The system is composed of a symmetric disk (the inertia wheel), which is attached at the end of a pendulum body. The disk is free to rotate about an axis parallel to the axis of rotation of the pendulum. This system is controlled by a DC motor actuating the inertia wheel and the coupling torque generated by the angular acceleration of the wheel.Two control approaches dedicated to stable limit cycle generation for the IWIP were presented by Andary et al. The first Andary et al. 2009a is based on partial nonlinear feedback linearization and dynamic control for optimal periodic reference trajectories tracking. The second [Andary et al., 2012] is a dual Model-free controller tracking some parameterized reference trajectories. Effectiveness of the two controllers was illustrated through numerical and experimental results on the IWIP testbed. The adjustment of the frequency and amplitude of the obtained limit cycles presents the limitation of this work. Therefore, some optimization problems must be solved to obtain reference trajectories with different periods. Many studies Fradkov \& Pogromsky, 1998, Marsden \& McCracken, 1976 dealt with useful self-oscillations emerging from Hopf bifurcation points.

Bifurcation analysis of the IWIP under control have been considered in the literature. Alonso et al. Alonso ${ }_{20}$ et al. 2005 used the bifurcation theory to classify different dynamical behaviors arising in the IWIP subject to bounded continuous state feedback control law. To limit the maximum amplitude of the control action, the control law is subject to a smooth saturation function, which was first introduced in Alonso et al. 2002 in order to stabilize the IWIP at the inverted position. The authors showed that the global dynamics of the IWIP change from a stable equilibrium point to a stable limit cycle via a Hopf bifurcation

25 as certain control gains change. A similar work was realized on the Furuta pendulum by analyzing the Hopf bifurcation [Pagano et al. 2000]. Recently, Nikolov and Nedev [Nikolov \& Nedev, 2016] analyzed bifurcation and dynamic behavior of the IWIP with bounded control by means of the theory of PoincaréAndronov-Hopf (PAH).

In this study we investigated the stabilization of oscillations by a passivity-based design control, namely

30 Interconnection and Damping assignment Passivity Based Control (IDA-PBC) introduced first in Ortega et al. 2002] and revisited in [Khraief-Haddad et al., 2014] and Khraief-Haddad et al. 2015]. The main contribution of the present work involves the tracking of reference trajectories (various stable limit cycles) from stabilization of the IWIP around equilibrium point by IDA-PBC controller. The IWIP transformed via IDA-PBC control into a generalized Hamiltonian closed-loop system exhibits critical Hopf bifurcation

35 points. Stable and unstable limit cycles emerge at these bifurcations. To study the direction and stability of such periodic solutions we proceeded to compute the first Lyapunov coefficient at the Hopf bifurcation. Practically, such solutions coincide with a great variety of vibrations (useful or destructive). At first, we analyzed the stability of the equilibrium point with respect to the control gains. Moreover, we developed conditions for the existence of the Hopf bifurcation and then of periodic oscillations. Furthermore, stabil-

40 ity of these periodic oscillations were investigated analytically by means of the first Lyapunov value as a function of some IDA-PBC control gains. The paper is organized as follows. In section 2 a brief description of the inertia wheel inverted pendulum and IDA-PBC controller is given. Section 3 deals with the Hopf-bifurcation analysis of the C-IWIP. The dynamical behaviour of the C-IWIP when Hopf bifurcation occurs is described in section 4. Analytical results about direction and stability of critical Hopf bifurcation

points are given in section 5. Section 6 is dedicated to simulation results. Finally, concluding remarks are drawn in Section 7.

\section{Controlled Inertia Wheel Inverted Pendulum (C-IWIP)}

\subsection{The inertia wheel inverted pendulum (IWIP)}

\subsubsection{Description}

${ }_{50}$ The underactuated system called IWIP, studied in this paper, is illustrated in figure(1). It has two degrees of freedom, the active one is the rotating disc and the passive joint is the pendulum. The generated torque produces an angular acceleration of the end mass which induces a coupling torque at the axis of 
the pendulum. The parameters of the system are summarized in (Table 1.).

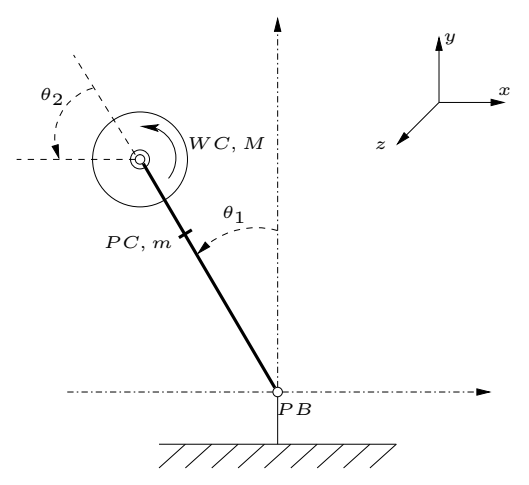

Figure 1. Inertia Wheel Inverted Pendulum.

Table 1.

Description of dynamical parameters of the IWIP

\begin{tabular}{|c|c|c|}
\hline Description & Notation & Units \\
\hline Pendulum angle with respect to vertical axis & $\theta_{1}$ & $\mathrm{rad}$ \\
\hline Wheel angle with respect to pendulum axis & $\theta_{2}$ & $\mathrm{rad}$ \\
\hline Mass of pendulum & $m$ & $k g$ \\
\hline Mass of the Wheel & $M$ & $\mathrm{~kg}$ \\
\hline $\begin{array}{l}\text { Length from pendulum base (PB) to pendulum center of mass } \\
\text { (PC) }\end{array}$ & $l$ & $m$ \\
\hline Length from pendulum base (PB) to Wheel center of mass (WC) & $L$ & $m$ \\
\hline $\begin{array}{l}\text { Rotational Inertia of pendulum about pendulum center of mass } \\
\text { (PC) }\end{array}$ & $I_{P C}$ & $\mathrm{~kg} \cdot \mathrm{m}^{2}$ \\
\hline Rotational Inertia of pendulum about pendulum base (PB) & $I_{P B}$ & $\mathrm{~kg} \cdot \mathrm{m}^{2}$ \\
\hline Rotational Inertia of Wheel about Wheel center of mass (WC) & $I_{W C}$ & $m^{2}$ \\
\hline Constant of gravitational acceleration & $g$ & $\mathrm{~m} / \mathrm{s}^{2}$ \\
\hline
\end{tabular}

\subsubsection{IWIP modelling}

${ }_{55}$ The Euler-Lagrange equations of motion can be written as [Spong \& Vidyasagar, 1989]:

$$
\left(\begin{array}{cc}
\left(a+I_{W C}\right) & I_{W C} \\
I_{W C} & I_{W C}
\end{array}\right)\left(\begin{array}{c}
\ddot{\theta}_{1} \\
\ddot{\theta_{2}}
\end{array}\right)-\left(\begin{array}{c}
b_{1} g \sin \theta_{1} \\
0
\end{array}\right)=\left(\begin{array}{l}
0 \\
u
\end{array}\right)
$$

where : $\theta=\left[\begin{array}{ll}\theta_{1} & \theta_{2}\end{array}\right]^{T}, u$ is the torque applied by the motor to spin the wheel and :

$$
\begin{aligned}
& a=M L^{2}+I_{P B} \\
& b_{1}=m l+M L .
\end{aligned}
$$

Let us introduce the following change of coordinates:

$$
\left[\begin{array}{l}
q_{1} \\
q_{2}
\end{array}\right]=\left[\begin{array}{ll}
1 & 0 \\
1 & 1
\end{array}\right]\left[\begin{array}{l}
\theta_{1} \\
\theta_{2}
\end{array}\right]
$$

This leads to a simplified model:

$$
\left[\begin{array}{cc}
a & 0 \\
0 & I_{W C}
\end{array}\right]\left[\begin{array}{c}
\ddot{q}_{1} \\
\ddot{q}_{2}
\end{array}\right]-\left[\begin{array}{c}
b_{1} g \sin q_{1} \\
0
\end{array}\right]=\left[\begin{array}{c}
-1 \\
1
\end{array}\right] u
$$


The model (4) can be written through Hamilton's equations of motion as Santibañez et al., 2005] Palacios, 60 2006:

$$
\left[\begin{array}{c}
\dot{q_{1}} \\
\dot{q_{2}} \\
\dot{p_{1}} \\
\dot{p_{2}}
\end{array}\right]=\left[\begin{array}{c}
\frac{p_{1}}{a} \\
\frac{p_{2}}{I_{W C}} \\
b_{1} g \sin q_{1}-u \\
u
\end{array}\right]
$$

Where $q=\left[\begin{array}{ll}q_{1} & q_{2}\end{array}\right]^{T}$ and $p=\left[\begin{array}{ll}p_{1} & p_{2}\end{array}\right]^{T}=\left[\begin{array}{ll}a \dot{q_{1}} & I_{W C} \dot{q}_{2}\end{array}\right]^{T}$ are the generalized positions and momenta, respectively. Which leads to write the Hamiltonian function as:

$$
H(q, p)=\frac{1}{2} p^{T} M^{-1}(q) p=\frac{1}{2}\left[\frac{p_{1}^{2}}{a}+\frac{p_{2}^{2}}{I_{W C}}\right]
$$

\subsection{Design of the control law for the IWIP}

\subsubsection{IDA-PBC controller}

${ }_{65}$ The control problem is to stabilize the pendulum at the upward position with the inertia disc aligned. We proposed to revisit the IDA-PBC control [Ortega et al. 2002]. The designed controller is formulated for port-controlled hamiltonian $(\mathrm{PCH})$ models. IDA-PBC aims to find a state feedback control $u$ such that the closed-loop dynamics is a port-hamiltonian system of the form:

$$
\dot{x}=\left[J_{d}(x, u)-R_{d}(x)\right] \frac{\partial H_{d}}{\partial x}(x)
$$

Where the new energy function $H_{d}$ has a local minimum at the desired equilibrium point. For the IWIP the desired hamiltonian function can be defined as:

$$
H_{d}(q, p)=\frac{1}{2} p^{T} M_{d}^{-1}(q) p+V_{d}(q)
$$

where $M_{d}=\left(\begin{array}{cc}a_{1} & a_{2} \\ a_{2} & a_{3}\end{array}\right)$ with $a_{1}>0, a_{1} a_{3}>a_{2}^{2}$ and $a_{1}+a_{2}<0$.

In Ortega et al. 2002 the authors propose the following control law for the IWIP:

$$
u=\gamma_{1} \sin \left(q_{1}\right)+k_{p}\left(q_{2}+\gamma_{2} q_{1}\right)+k_{v} k_{2}\left(\dot{q_{2}}+\gamma_{2} \dot{q}_{1}\right)
$$

where

$$
\begin{aligned}
& \gamma_{1}>b_{1} g \\
& \gamma_{2}=-\frac{a \gamma_{1}}{I_{W} C\left(\gamma_{1}-b_{1}\right)} \\
& k_{2}>0
\end{aligned}
$$

Together with $k_{p}$ and $k_{v}$ positive arbitrary constants, define the tuning gains.

The control law (9) can be rewritten Khraief-Haddad et al. 2014 and Khraief-Haddad et al., 2015] in 75 terms of the generalized coordinates $q$ and momenta $p$ as

$$
u=\underbrace{\gamma_{1} \sin \left(q_{1}\right)+k_{1} q_{1}+k_{2} q_{2}}_{u_{e s}}+\underbrace{k_{3} p_{1}+k_{4} p_{2}}_{u_{d i}}
$$

where $u_{e s}$ is the energy shaping control to assign the equilibrium and $u_{d i}$ injects damping to achieve asymptotic stability.

$$
\begin{aligned}
& k_{1}=k_{p} \gamma_{2} \\
& k_{2}=k_{p} \\
& k_{3}=k_{v}\left(\frac{a_{2}+a_{3}}{a_{1} a_{3}-a_{2}^{2}}\right) \\
& k_{4}=-k_{v}\left(\frac{a_{1}+a_{2}}{a_{1} a_{3}-a_{2}^{2}}\right)
\end{aligned}
$$




\subsubsection{Simulation results}

Table 2 .

Dynamic Parameters of the IWIP

\begin{tabular}{|l|c||c|c|}
\hline Parameter & Description & Value & Unit \\
\hline$m$ & pendulum mass & 3.228 & $\mathrm{Kg}$ \\
$M$ & mass of the wheel & 0.33081 & $\mathrm{Kg}$ \\
$l$ & Pendulum center of mass position & 0.06 & $\mathrm{~m}$ \\
$L$ & Wheel center of mass position & 0.044 & $\mathrm{~m}$ \\
$I_{1}$ & Pendulum inertia & 0.0314 & $\mathrm{Kgm}^{2}$ \\
$I_{2}$ & Wheel inertia & $4.176 e^{-4}$ & $\mathrm{Kgm}^{2}$ \\
\hline
\end{tabular}

In the simulations below we considered the $\mathrm{PCH}$ model(5). IWIP parameters are described in table 2. They were identified on the real prototype of the system Andary et al. 2009b], which was designed and developed at LIRMM 1 1 . Here after, we will present and discuss simulation results obtained on the IWIP when applying IDA-PBC control law (10). We chose the initial configuration $\left[\begin{array}{llll}q_{1} & q_{2} & p_{1} & p_{2}\end{array}\right]^{T}=\left(\begin{array}{llll}0.2 & 0 & 0 & 0\end{array}\right)^{T}$. The remaining control parameters were selected as $\gamma_{1}=6.1284$, $k_{1}=1.0367$ and $k_{2}=0.0011 . k_{3}=16.62$ and $k_{4}=3.4640$.

Figure(2) depicts joint positions and velocities and also displays the control input. The performance of the IDA-PBC controller was observed by the convergence towards the equilibrium point $(0,0,0,0)^{T}$.
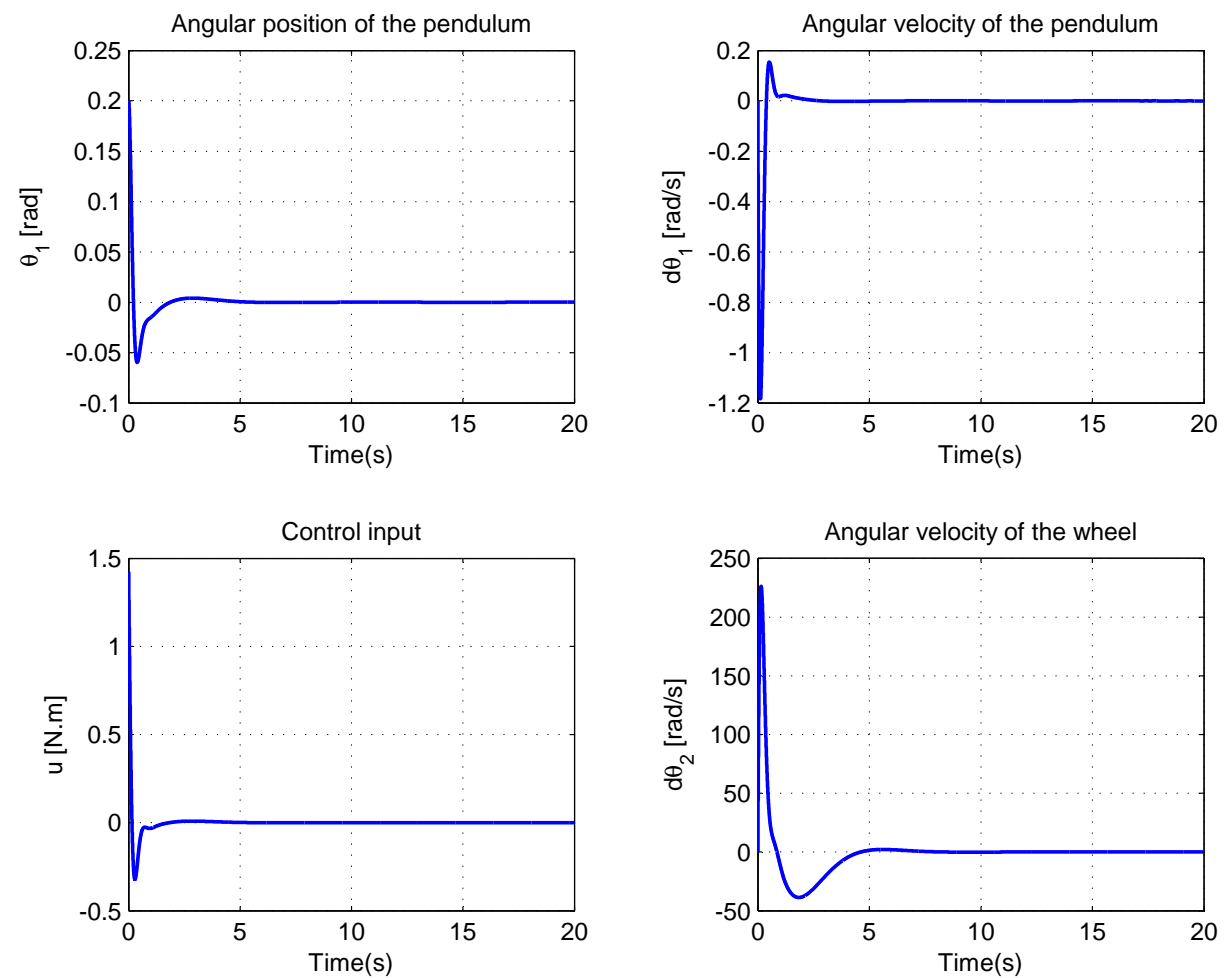

Figure 2. Simulation results in the nominal case

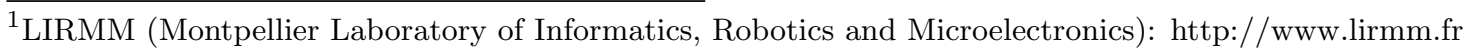




\section{Hopf Bifurcation analysis of the C-IWIP}

\subsection{Equilibrium point}

The equilibrium point of the closed loop system (5) is determined when $\dot{q}_{1}=0, \dot{q_{2}}=0, \dot{p_{1}}=0, \dot{p_{2}}=0$ and thus:

$$
\left\{\begin{array}{c}
p_{1}=0 \\
p_{2}=0 \\
\sin q_{1}=0 \\
k_{1} q_{1}+k_{2} q_{2}=0
\end{array}\right.
$$

Therefore, the equilibrium point of the system (5) is given by:

$$
\boldsymbol{x}_{e q}=\left[\begin{array}{c}
k \pi \\
-k \pi \frac{k_{1}}{k_{2}} \\
0 \\
0
\end{array}\right]
$$

90 So we can distinguish $x_{e q}=\left(2 k \pi,-2 k \pi \frac{k_{1}}{k_{2}}, 0,0\right)$ (inverted or upward position) or $x_{e q}=((2 k+1) \pi,-(2 k+$ 1) $\left.\pi \frac{k_{1}}{k_{2}}, 0,0\right)$ (hanging or rest position), $k \in \mathbb{Z}$. To avoid considering an infinite number of equilibria, we dealt only with one equilibrium point $x_{e q}=(0,0,0,0)^{T}$ stabilized by the IDA-PBC control and corresponding to the pendulum at the inverted position with zero disc velocity.

\subsection{Existence of the Hopf Bifurcation}

${ }_{95}$ Let $X=(q, p)^{T}$, and $p=\left(k_{1}, k_{2}, k_{3}, k_{4}\right)^{T}$. Recall $(5)$ which we restate here:

$$
\dot{X}=F(X, p), \quad X \in \mathbb{R}^{4}, \quad p \in \mathbb{R}^{4}
$$

Equation (13) represents the vector field of the C-IWIP.

\subsubsection{Jacobian Matrix}

The Jacobian matrix $\mathcal{J}$ around the equilibrium point $\boldsymbol{x}_{e q}$ of the closed-loop system (5) is defined as follows:

$$
\mathcal{J}=\left[\begin{array}{cccc}
0 & 0 & \frac{1}{a} & 0 \\
0 & 0 & 0 & \frac{1}{I_{w c}} \\
b_{1} g-\gamma_{1}-k_{1} & -k_{2} & -k_{3} & -k_{4} \\
\gamma_{1}+k_{1} & k_{2} & k_{3} & k_{4}
\end{array}\right]
$$

Let us make a variable change as: $a=\frac{1}{a}, c=\frac{1}{I_{w c}}, b=b_{1} g$ and $\gamma=\gamma_{1}$. Then, we obtain:

$$
\mathcal{J}=\left[\begin{array}{cccc}
0 & 0 & a & 0 \\
0 & 0 & 0 & c \\
b-\gamma-k_{1} & -k_{2} & -k_{3} & -k_{4} \\
\gamma+k_{1} & k_{2} & k_{3} & k_{4}
\end{array}\right]
$$

\subsubsection{Eigenvalues of the Jacobian Matrix}

The eigenvalues of the Jacobian matrix $\mathcal{J}$ (15) are computed by solving the following equation

$$
\operatorname{det}(\lambda(p) \mathcal{I}-\mathcal{J})=0
$$


It is easy to demonstrate that relation $(16)$ can be reformulated as follows:

$$
\lambda(p)^{4}+\left(k_{3}-k_{4}\right) \lambda(p)^{3}+\left(a\left(\gamma-b+k_{1}\right)-c k_{2}\right) \lambda(p)^{2}+a b k_{4} \lambda(p)+a b c k_{2}=0
$$

We assume that the Jacobian matrix $\mathcal{J}$ admits two complex-conjugate eigenvalues, $\alpha(p) \pm i \beta(p)$ where $\beta(p)>0$, and two eigenvalues $\mu$ and $\eta$ verifying : $(\mu+\eta)<0$ and $(\mu \eta)>0$ and are both real. Thus, the characteristic polynomial can be expressed as

$$
(\lambda(p)-\mu)(\lambda(p)-\eta)(\lambda(p)-(\alpha(p)+i \beta(p)))(\lambda(p)-(\alpha(p)-i \beta(p)))=0
$$

This last relation can be cast as:

$$
\begin{aligned}
& \lambda(p)^{4}+[-2 \alpha(p)-(\mu+\eta)] \lambda(p)^{3}+\left[\left(\alpha(p)^{2}+\beta(p)^{2}\right)+2 \alpha(p)(\mu+\eta)+\mu \eta\right] \lambda(p)^{2} \\
& +\left[-(\mu+\eta)\left(\alpha(p)^{2}+\beta(p)^{2}\right)-2 \alpha(p) \mu \eta\right] \lambda(p)+\mu \eta\left(\alpha(p)^{2}+\beta(p)^{2}\right)=0
\end{aligned}
$$

According to expressions (17) and $(19)$ we can write 2 ,

$$
\begin{gathered}
-2 \alpha-(\mu+\eta)=k_{3}-k_{4} \\
\left(\alpha^{2}+\beta^{2}\right)+2 \alpha(\mu+\eta)+\mu \eta=a\left(\gamma-b+k_{1}\right)-c k_{2}
\end{gathered}
$$

$$
\begin{gathered}
-(\mu+\eta)\left(\alpha^{2}+\beta^{2}\right)-2 \alpha \mu \eta=a b k_{4} \\
\mu \eta\left(\alpha^{2}+\beta^{2}\right)=a b c k_{2}
\end{gathered}
$$

\subsubsection{The Hopf bifurcation}

The Hopf bifurcation occurs if and only if $\alpha(p)=0$ and $\beta(p) \neq 0$. Then, relations in $(20)$ can be simplified as follows:

$$
\mu+\eta=k_{4}-k_{3}
$$

115

$$
\begin{gathered}
\beta^{2}+\mu \eta=a\left(\gamma-b+k_{1}\right)-c k_{2} \\
-(\mu+\eta) \beta^{2}=a b k_{4} \\
\mu \eta \beta^{2}=a b c k_{2}
\end{gathered}
$$

The problem was then to find the gain parameters, $k_{i}$, for which the Hopf bifurcation happens.

The functions in 21] can be reformulated as

$$
\mu+\eta=k_{4}-k_{3}
$$

120

$$
\beta^{4}-\left(a\left(\gamma-b+k_{1}\right)-c k_{2}\right) \beta^{2}+a b c k_{2}=0
$$

\footnotetext{
${ }^{2}$ In the following we take $\beta(p)=\beta$
} 


$$
\begin{gathered}
k_{4}=\frac{\beta^{2}}{a b+\beta^{2}} k_{3} \\
\mu \eta=\frac{a b c}{\beta^{2}} k_{2}
\end{gathered}
$$

Using relation 22c in 22a and relying on expression (22d), we emphasize that: $\mu+\eta<0$ and $\mu \eta>0$.

Furthermore, relying on the previous relations in $(22)$, it is worth noting that $\beta$ is the solution of (22b) and it depends only on the two parameters $k_{1}$ and $k_{2}$. Moreover, $k_{4}$ is proportional to $k_{3}$ and it depends on $\beta$. Then, by choosing the value of $k_{1}, k_{2}$ and $k_{3}$, we can calculate the value of the $k_{4}$ at which the Hopf bifurcation occurs. We state that the gain $k_{3}$ does not affect the occurrence of the HB.

By solving relation (22b), it is easy to show that there are two solutions $\beta_{1}$ and $\beta_{2}$ defined as follows:

$$
\beta_{1}=\sqrt{\frac{a\left(\gamma-b+k_{1}\right)-c k_{2}-\sqrt{\left(a\left(\gamma-b+k_{1}\right)-c k_{2}\right)^{2}-4 a b c k_{2}}}{2}}
$$

$$
\beta_{2}=\sqrt{\frac{a\left(\gamma-b+k_{1}\right)-c k_{2}+\sqrt{\left(a\left(\gamma-b+k_{1}\right)-c k_{2}\right)^{2}-4 a b c k_{2}}}{2}}
$$

In (23), we have $\beta_{1}<\beta_{2}$. In fact, both solutions, $\beta_{1}$ and $\beta_{2}$, exist if and only if the following three conditions are met:

$$
\begin{gathered}
\left(a\left(\gamma-b+k_{1}\right)-c k_{2}\right)^{2}-4 a b c k_{2}>0 \\
a\left(\gamma-b+k_{1}\right)-c k_{2}-\sqrt{\left(a\left(\gamma-b+k_{1}\right)-c k_{2}\right)^{2}-4 a b c k_{2}}>0 \\
a\left(\gamma-b+k_{1}\right)-c k_{2}+\sqrt{\left(a\left(\gamma-b+k_{1}\right)-c k_{2}\right)^{2}-4 a b c k_{2}}>0
\end{gathered}
$$

Proposition 3.1. Consider the C-IWIP represented by the vector field (13). According to conditions (24b) and (24c) and as $k_{1}>0$ and $k_{2}>0$, it follows that: $a\left(\gamma-b+k_{1}\right)-c k_{2}>0$. Hence, relying on 24a), it is easy to show that, for some $k_{2}>0$, we have:

$$
k_{1}>b-\gamma+\frac{c}{a} k_{2}+2 \sqrt{\frac{b c}{a}} \sqrt{k_{2}}
$$

Expression (25) represents the condition on $k_{1}$ with respect to $k_{2}$ for the existence of the Hopf bifurcation for the $C-I W I P$.

\section{Dynamical behaviour of the C-IWIP when Hopf bifurcation occurs}

In this section we are interested in analyzing the dynamical behaviour of the C-IWIP near the equilibrium point $x_{e q}$ when the controlled parameters represented by $p$ are varied.

Fig. 3 illustrates such conditions in the parameters space $\left(k_{1}, k_{2}\right)$. HB denotes the zone in which the two

Hopf bifurcations (HB) occur. In the white space, the two HB disappear simultaneously. This type of 
curve is known as the failure of the transversality condition of the eigenvalue crossing of the regular Hopf bifurcation. The BC-HB represents a degenerate Hopf bifurcation and is defined by the following equation

$$
k_{1, c}=b-\gamma+\frac{c}{a} k_{2}+2 \sqrt{\frac{b c}{a}} \sqrt{k_{2}}
$$

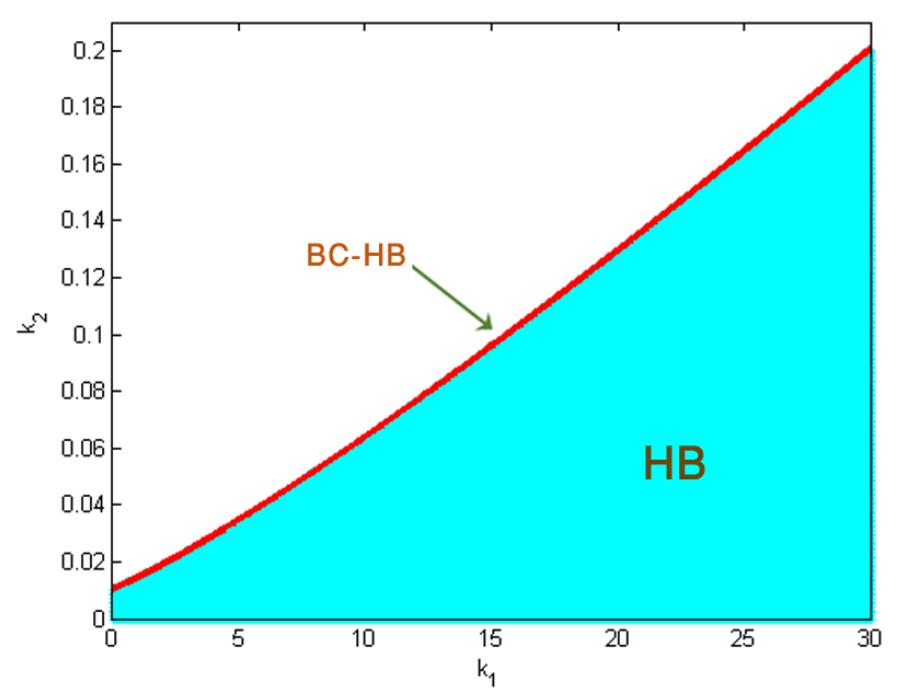

Figure 3. Existence of the Hopf Bifurcation (HB) in the parameters space $\left(k_{1}, k_{2}\right)$.

Hence, for such expression (26), we obtain: $\beta_{1}=\beta_{2}=\beta_{c}$

$$
\beta_{c}=\sqrt{\sqrt{a b c} \sqrt{k_{2}}}
$$

Then, the two Hopf bifurcations enter in collision and are replaced by only one Hopf bifurcation. Accordingly, The BC-HB reveals the curve for which only one Hopf exists. Varying one parameter $k_{1}$ or $k_{2}$ or the two parameters together provokes the disappearance of these two HBs and the birth of only one HB. Thus, slightly varying these two parameters, the HB disappears and thus HB no longer exists.

Fig. 4 shows the variation of $\beta_{1}$ and $\beta_{2}$ in (23) as the gain $k_{1}$ varies. Here, we chose 5 different fixed values of the gain $k_{2}: k_{2}=0.001, k_{2}=0.02, k_{2}=0.05, k_{2}=0.1$ and $k_{2}=0.15$. Small circles show the critical value at which only one HB occurs. The value of $\beta$ is given by expression (27). It is obvious that for some fixed value of $k_{2}$, and as $k_{1}$ decreases, the two HBs meet and annihilate with each other at the critical value $k_{1, c}$ given via relation (26). Hence by slightly decreasing $k_{1}$, the HB disappears suddenly. Relying on Fig. 4 , for $k_{2}=0.001$ and as $k_{1}>0$, the two HBs always coexist.

We dealt with the variation of the gain $k_{4}$ as $k_{1}$ varied for a constant value of $k_{3}$ and for different values of $k_{2}$. Such investigation was realized by means of relation $\left.22 \mathrm{c}\right)$. Let $k_{3}=38.4$. Fig. 5 shows the variation of $k_{4}$ with respect to $k_{1}$ for some values of $k_{2}$.

For example, for $k_{1}=k_{p} * \gamma_{2}=1.036691874482759, k_{2}=k_{p}=0.0011$ and $k_{3}=38.4$, the two values of $k_{4}$ were calculated to be about: $k_{4,1}=0.871599173381224$ and $k_{4,2}=27.197725293683821$.

\section{Direction and stability of the Hopf bifurcation}

\subsection{Analytical analysis}

In the previous section, we obtained some conditions which guarantee that the C-IWIP undergoes Hopf bifurcation at the equilibrium point $x_{e q}$. In this section we will use the normal form and the center manifold theory Kuznetsov, 1998, Wiggins, 2003] and Guckenheimer \& Holmes, 1983 to give the explicit formulae 


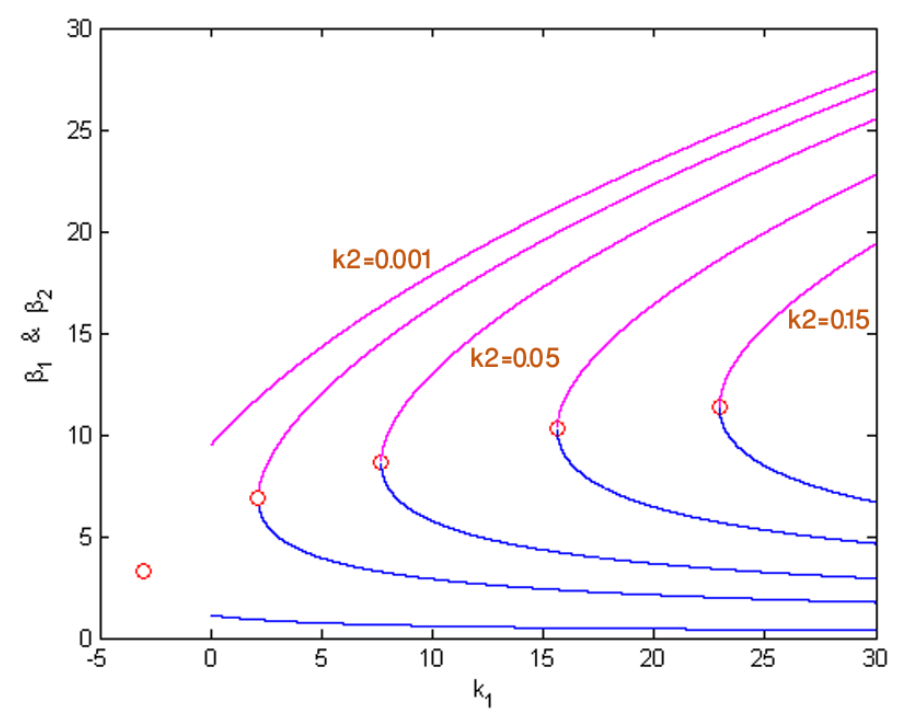

Figure 4. Variation of $\beta_{1}$ and $\beta_{2}$ with respect to parameter $k_{1}$ for fixed values of $k_{2}$.

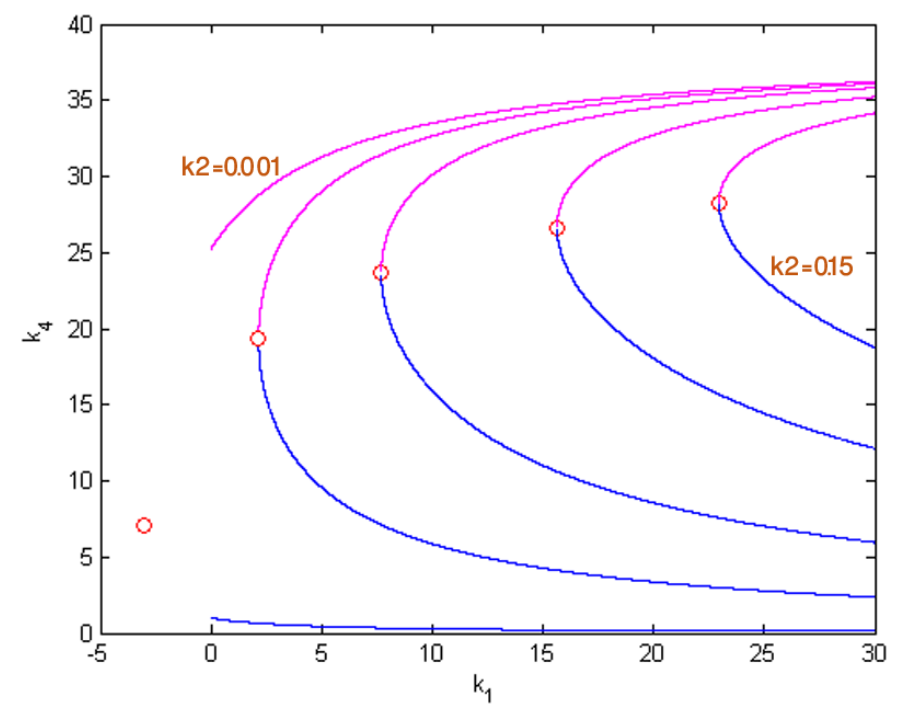

Figure 5. Variation of $k_{4}$ with respect to parameter $k_{1}$, for $k_{3}=38.4$ and for fixed values of $k_{2}: k_{2}=0.001, k_{2}=0.02$, $k_{2}=0.05, k_{2}=0.1$ and $k_{2}=0.15$.

which determine the direction and stability of the periodic solutions bifurcating from equilibrium point $x_{e q}$ 170 at some critical values of the controlled parameters denoted by vector $p$. In what follows we will assume that we are dealing with a single scalar parameter, i.e $p=k_{2}$. We fix $k_{1}=k_{1, c}(26)$ and leave $k_{2}$ free to vary. In this case $\beta^{2}=\beta_{c}^{2}=\sqrt{a b c k_{2}}$, so we deal only with one Hopf bifurcation. According to expressions (21), 22), 26) and (27) we write the folloing expressions:

$$
\begin{gathered}
k_{1}=b-\gamma+\frac{\beta^{4}}{a^{2} b}+2 \frac{\beta^{2}}{a} \\
k_{2}=\frac{\beta^{4}}{a b c}
\end{gathered}
$$

$$
k_{3}=-(\mu+\eta)\left(1+\frac{\beta^{2}}{a b}\right)
$$




$$
k_{4}=-(\mu+\eta)\left(\frac{\beta^{2}}{a b}\right)
$$

The jacobian matrix 15 evaluated at $x_{e q}$ can be rewritten as follows:

$$
\mathcal{J}=\left[\begin{array}{cccc}
0 & 0 & a & 0 \\
0 & 0 & 0 & c \\
-\frac{\beta^{4}}{a^{2} b}-2 \frac{\beta^{2}}{a} & -\frac{\beta^{4}}{a b c} & (\mu+\eta)\left(1+\frac{\beta^{2}}{a b}\right) & (\mu+\eta)\left(\frac{\beta^{2}}{a b}\right) \\
b+\frac{\beta^{4}}{a^{2} b}+2 \frac{\beta^{2}}{a} & \frac{\beta^{4}}{a b c} & -(\mu+\eta)\left(1+\frac{\beta^{2}}{a b}\right) & -(\mu+\eta)\left(\frac{\beta^{2}}{a b}\right)
\end{array}\right]
$$

Whether Hopf bifurcation is subcritical or supercritical, it is determined by the sign of the first Lyapunov coefficient $l_{1}(0)$ of the C-IWIP near the equilibrium point. In what follows, we will adopt the convention of Kuznetsov [Kuznetsov, 1998] and Kuehn, 2010] to compute $l_{1}(0)$.

Suppose the system (13) is written as:

$$
\dot{X}=\mathcal{J} X+f(X)
$$

Where $f(X)=O\left(\left\|X^{2}\right\|\right)$ is a smooth function. Write the Taylor expansion to transform (30)into the following normal form:

$$
\dot{X}=\mathcal{J} X+\frac{1}{2} B(X, X)+\frac{1}{6} C(X, X, X)+O\left(\left\|X^{4}\right\|\right)
$$

Where $B(u, v)$ and $C(u, v, w)$ are multilinear functions with the following components:

$$
\begin{gathered}
B_{i}(u, v)=\left.\sum_{j, k=1}^{4} \frac{\partial^{2} f_{i}(\xi)}{\partial \xi_{j} \partial \xi_{k}}\right|_{\xi=0} u_{j} v_{k} \\
C_{i}(u, v, w)=\left.\sum_{j, k, l=1}^{4} \frac{\partial^{3} f_{i}(\xi)}{\partial \xi_{j} \partial \xi_{k} \partial \xi_{l}}\right|_{\xi=0} u_{j} v_{k} w_{l}
\end{gathered}
$$

We can check easily that for the C-IWIP equation 30 is given by:

$$
\dot{X}=\left[\begin{array}{cccc}
0 & 0 & a & 0 \\
0 & 0 & 0 & c \\
b-\gamma-k_{1} & -k_{2} & -k_{3} & -k_{4} \\
\gamma+k_{1} & k_{2} & k_{3} & k_{4}
\end{array}\right] X+\left[\begin{array}{c}
0 \\
0 \\
(b-\gamma) \frac{q_{1}^{3}}{6} \\
-\gamma \frac{q_{1}^{3}}{6}
\end{array}\right]+0\left(\left\|X^{4}\right\|\right)
$$

We can verify also that the bilinear function $B(u, v)=0$ while the multilinear function $C(u, v, w)$ can be expressed as :

$$
C(u, v, w)=\left[\begin{array}{c}
0 \\
0 \\
(b-\gamma) u_{1} v_{1} w_{1} \\
-\gamma u_{1} v_{1} w_{1}
\end{array}\right]
$$

The matrix $\mathcal{J}$ has eigenvalues : $\lambda_{1,2}= \pm i \beta$ for $\beta>0, \lambda_{3}=\mu$ and $\lambda_{4}=\eta$. Let $q \in \mathbb{C}^{4}$ be the eigenvector of $\lambda_{1}$ and $p \in \mathbb{C}^{4}$ the corresponding eigenvector of the transpose $\mathcal{J}^{T}$ i.e:

$\mathcal{J} q=i \beta q ; \mathcal{J} \bar{q}=-i \beta \bar{q}$ and $\mathcal{J}^{T} p=-i \beta p ; \mathcal{J}^{T} \bar{p}=i \beta \bar{p}$. It is easy to check (with mupad/Matlab) that the vectors :

$$
q=\left(\begin{array}{c}
\frac{a \beta \mathrm{i}}{a b+\beta^{2}} \\
\frac{-c \mathrm{i}}{\beta} \\
\frac{-\beta^{2}}{a b+\beta^{2}} \\
1
\end{array}\right)
$$




$$
p=\left(\begin{array}{c}
-\frac{\left(\beta^{2}+a b\right)\left(\beta^{3}+a b \beta-a b \eta \mathrm{i}-a b \mu \mathrm{i}\right)}{2 a^{2} b \beta(\mu+\eta)} \\
-\frac{\beta^{4}+a b \beta^{2}}{2 a b c(\mu+\eta)} \\
-\frac{\left(\beta^{2}+a b\right)\left(a b \mathrm{i}-\mu \beta-\mathrm{nu} \beta+\beta^{2} \mathrm{i}\right)}{2 a b \beta(\mu+\eta)} \\
\frac{\left(\beta^{2}+a b\right)(\mu+\eta-\beta \mathrm{i})}{2 a b(\mu+\eta)}
\end{array}\right)
$$

are eigenvectors of $\mathcal{J}$ and $\mathcal{J}^{T}$ corresponding to respectively the eigenvalues $i \beta$ and $-i \beta$. Note that the $p$ is normalized so that the standard complex inner product with $q$ satisfies: $\bar{p}^{T} q=\sum_{j=1}^{4} \bar{p}_{j}^{T} q_{j}=1$.

The first Lyapunov coefficient of the Hopf bifurcation is defined by [Kuznetsov, 1998]:

$$
l_{1}(0)=\frac{1}{2 \beta}\left(\bar{p}^{T} C(q, q, \bar{q})-2 \bar{p}^{T} B\left(q, \mathcal{J}^{-1} B(q, \bar{q})\right)+\bar{p}^{T} B\left(\bar{q},\left(2 i \beta I_{4}-\mathcal{J}\right)^{-1} B(q, q)\right)\right)
$$

Using expressions of $q, p$ in (35) and $C(u, v, w)$ in (34) and relying on (36) we obtained the first Lyapunov coefficient of the C-IWIP for the considered Hopf bifurcation point as follows:

$$
\begin{gathered}
l_{1}(0)=\frac{1}{2 \beta} \operatorname{Re}\left[\bar{p}^{T} C(q, q, \bar{q})\right] \\
l_{1}(0)=\frac{130.7340692 \beta\left(\beta^{2}-93.42868368\right)}{(\mu+\eta)\left(\beta^{2}+46.71434184\right)^{2}}
\end{gathered}
$$

We set $\varsigma=93.42868368$.

Proposition 5.1. Consider the C-IWIP represented by the vector field (13). According to the expression of the first Lyapunov coefficient (37b) the Hopf bifurcation (in the case where $\beta=\beta_{c}$ ) is non degenerate and supercritical if and only if $\beta^{2}>\varsigma$.

We can now return to the IDA-PBC control parameters by making some substitutions and met the following result:

Proposition 5.2. For all initial conditions -except a set of zero measures- the IDA-PBC drives the IWIP

towards a stable limit cycle with the period $\frac{2 \pi}{\beta}$ if control parameters satisfy the following conditions:

$$
\begin{gathered}
k_{1}>b-\gamma+\frac{\varsigma^{2}}{a^{2} b}+2 \frac{\varsigma^{2}}{a} \\
k_{2}>\frac{\varsigma^{2}}{a b c} \\
\frac{k_{4}}{k_{3}}>\frac{\varsigma}{\varsigma+a b}
\end{gathered}
$$

\subsection{IDA-PBC controller gains and IWIP stable oscillations}

In this section, we will perform some graphical illustrations of the boundary stability with respect to IDAPBC controller gains. The control objective was to obtain stable and robust oscillations for the IWIP. Thus, the region defined by (38) with respect to the first Lyapunov coefficient is depicted in (6). Here we note that we did simulations when we take into account the assumption $\mu+\nu<0$. It is seen that Hopf bifurcation is supercritical when $l_{1}(0) \leq 0$ or $\beta>\sqrt{\varsigma}=9.66$. 

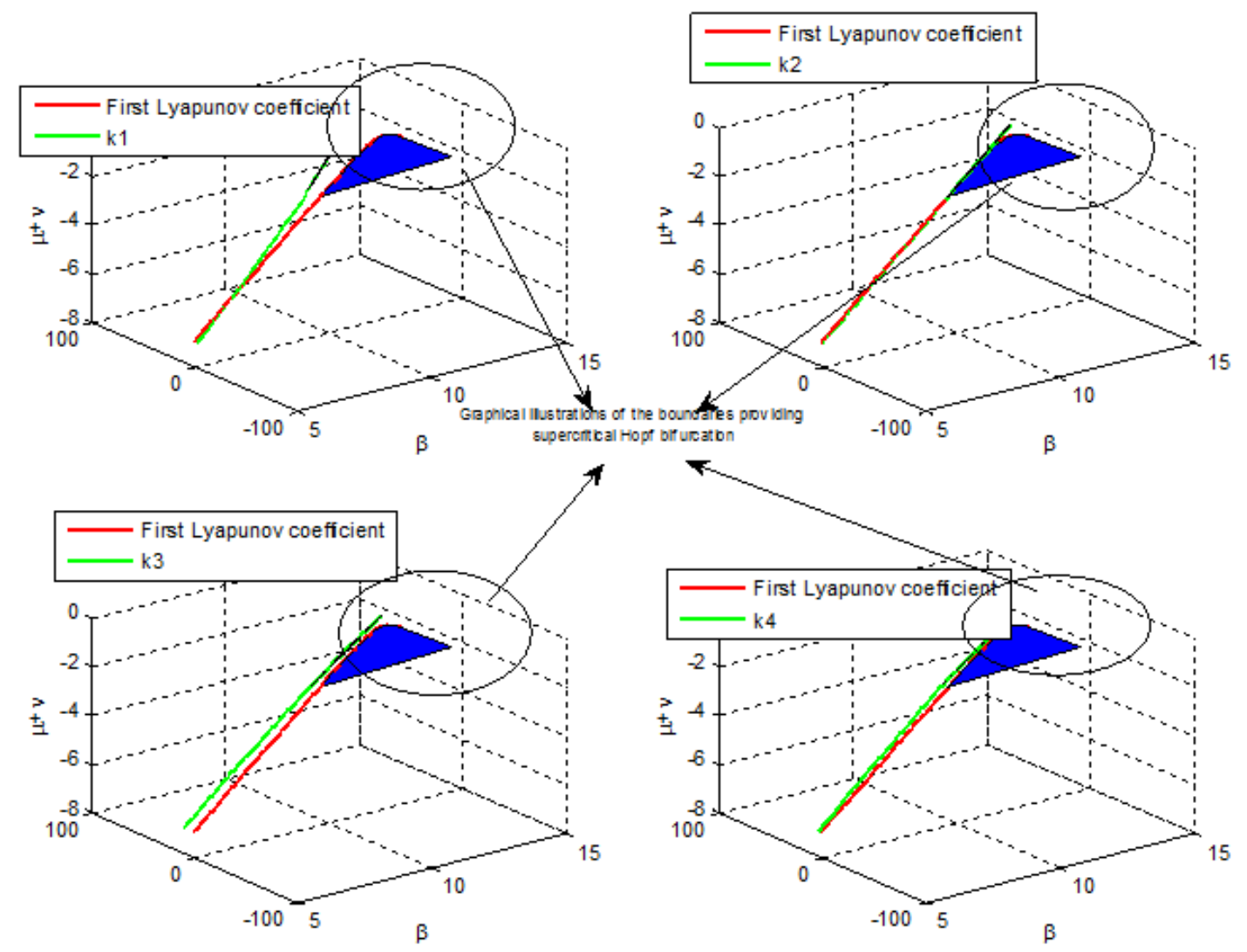

Figure 6. Graphical illustration of supercritical Hopf bifurcation zones with respect to parameters $k_{1}, k_{2}, k_{3}$ and $k_{4}$

\section{Numerical Simulation}

Some numerical Hopf bifurcation analysises of the C-IWIP were carried out to attest the effectiveness of the analytical results obtained in the previous section. We performed a numerical computation of Hopf bifurcation points and the first Lyapunov coefficient $l_{1}(0)$, using the well-known MATCONT package Dhooge et al. 2003 which used the AUTO package to the numerical continuation of limit cycles and the stability index.

\subsection{Hopf bifurcation detection for the C-IWIP}

This study dealt with one-parameter bifurcation analysis of equilibria, thus we fixed three parameters and varied only one. For example for $k_{2}=0.0782, k_{3}=4.94$ and $k_{4}=3.4640$ we found only one critical value of $k_{1,1}^{c}=12.386476$ at which the Hopf bifurcation occured (see figure(7)). Moreover, we had $\mu=-0.738041+i 8.90205, \eta=-0.738041-i 8.90205, l 1(0)=-64.76669 e-2$ and $\beta=10.4705$. We proved that the previous results coincide with conditions met in proposition 5.1 and 5.2 . Then we choose $k_{2}, k_{3}$ and $k_{4}$ satisfying conditions (38). Here, we took $k_{2}=0.0783$ and fixed $k_{3}=38.4$ and $k_{4}=35.97$. By varying the parameter $k_{1}$ we found one critical value of $k_{1,2}^{c}=34.905957$ at which the Hopf bifurcation occured (see figure (8)). Tables 3. and 4. show a comparison between numerical and analytical results for both critical points $\left(k_{1,1}^{c}\right.$ and $\left.k_{1,2}^{c}\right)$. It can be shown that both results coincide. Moreover, all values attain assumptions met in subsection 5.1. The difference between each cycle is the amplitude and the frequency. Relying on proposition 5.1 the period of the stable limit cycles around the critical parameters $k_{1,1}^{c}$ and $k_{1,2}^{c}$ were respectively : $T_{1,1}^{c}=0.6 s$ and $T_{1,2}^{c}=0.2389 \mathrm{~s}$. 


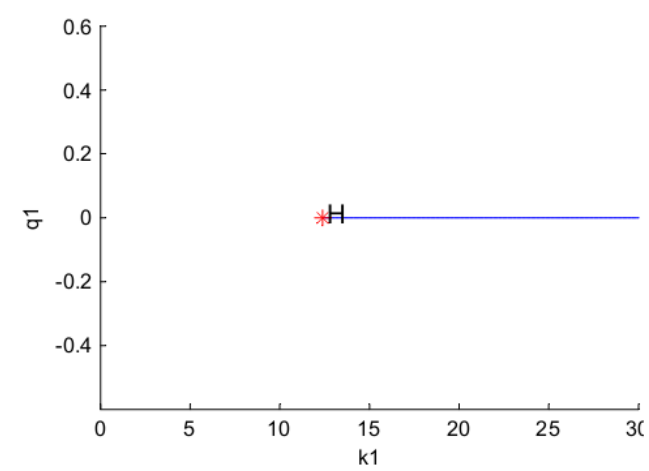

Figure 7. Hopf bifurcation detection with respect to parameter $k_{1}$ for fixed values of $k_{2}, k_{3}$ and $k_{4}$

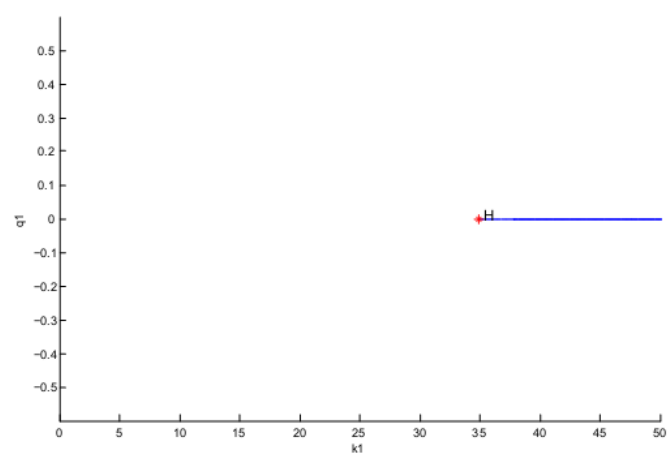

Figure 8. Hopf bifurcation detection with respect to parameter $k_{4}$ for fixed values of $k_{1}, k_{2}$ and $k_{3}$

Table 3.

Numerical and analytical values for critical points when $k_{1}=k_{1,1}^{c}$

\begin{tabular}{|l|c||c|c|}
\hline Parameter & Description & Numerical results & analytical results \\
\hline$l_{1}(0)$ & The first Lyapunov coefficient & $-64.2935 e-2$ & -0.6147 \\
$\lambda_{1}$ & Eigenvalue 1 & $-0.738041+i 8.90205$ & $\lambda_{1}+\lambda_{2}<0$ \\
$\lambda_{2}$ & Eigenvalue 2 & $-0.738041-i 8.90205$ & $\lambda_{1}+\lambda_{2}<0$ \\
$\lambda_{3}$ & Eigenvalue 3 & $i 10.4705=i \beta$ & $\beta^{2}>\alpha$ \\
$\lambda_{4}$ & Eigenvalue 4 & $-i 10.4705=-i \beta$ & $\beta^{2}>\alpha$ \\
\hline
\end{tabular}

Table 4.

Numerical and analytical values for critical points when $k_{1}=k_{1,2}^{c}$

\begin{tabular}{|c|c|c|c|}
\hline Parameter & Description & Numerical results & analytical results \\
\hline$l_{1}(0)$ & The first Lyapunov coefficient & $-15.2935 e-2$ & -1.5526 \\
\hline$\lambda_{1}$ & Eigenvalue 1 & $-1.215+i 3.3452$ & $\lambda_{1}+\lambda_{2}<0$ \\
\hline$\lambda_{2}$ & Eigenvalue 2 & $-1.215-i 3.3452$ & $\lambda_{1}+\lambda_{2}<0$ \\
\hline$\lambda_{3}$ & Eigenvalue 3 & $i 26.2961=i \beta$ & $\beta^{2}>\alpha$ \\
\hline$\lambda_{4}$ & Eigenvalue 4 & $-i 26.2961=-i \beta$ & $\beta^{2}>\alpha$ \\
\hline
\end{tabular}




\subsection{Stable Limit Cycle generation of the IWIP via IDA-PBC}

\subsubsection{Case $1: k_{1}=k_{1,1}^{c}$}

In this first case, uncertainties and external disturbances were neglected. The proposed simulation is from the initial condition given in Table 5 . Then the application of the control law 10 to the nominal nonlinear dynamics of the IWIP gave results shown in figures $(9)$ and $(10)$. The former displays the pendulum joint position and velocity versus time. These results clearly show the convergence of the trajectory of the controlled IWIP to the desired stable period-1 limit cycle. It can be observed from the displayed results that the period of the reference limit cycle is about $0.6207[s]$. The latter represents the

Table 5 .

Description and Numerical values for critical points

\begin{tabular}{|l|c||c|c|}
\hline Parameter & Description & $k_{1,1}^{c}$ & $k_{1,2}^{c}$ \\
\hline$l_{1}(0)$ & The first Lyapunov coefficient & -0.6147 & -1.5526 \\
$X_{0}$ & Initial value & {$[0.2 ; 0 ; 0 ; 0]$} & {$[0.2 ; 0 ; 0 ; 0]$} \\
$k_{1}$ & Control parameter 1 & 12.386476 & 34.905957 \\
$k_{2}$ & Control parameter 2 & 0.0782 & 0.0783 \\
$k_{3}$ & Control parameter 3 & 4.94 & 38.4 \\
$k_{4}$ & Control parameter 4 & 3.4640 & 35.972012 \\
\hline
\end{tabular}

limit cycle.
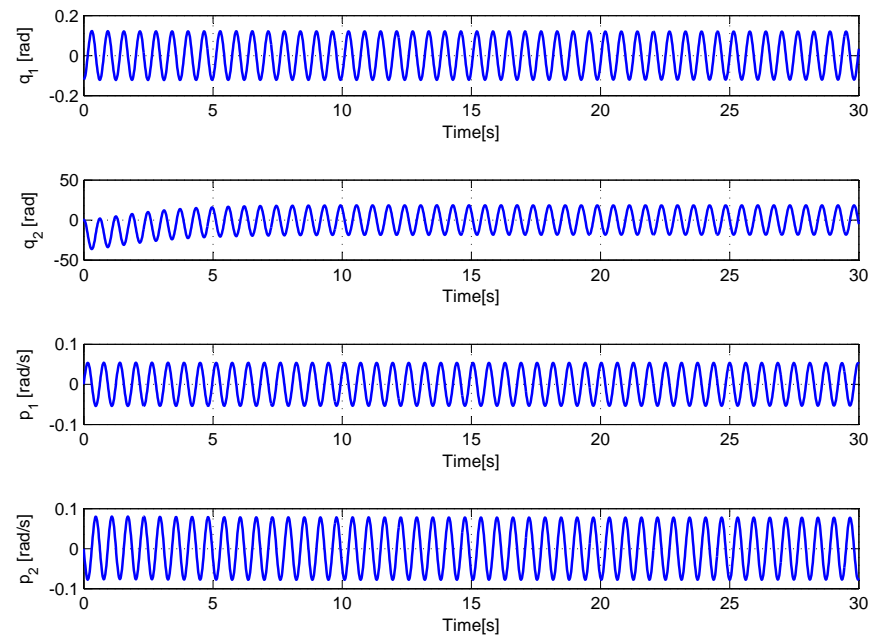

Figure 9. Pendulum joint position and velocity versus time (Initial conditions are $[0.2 ; 0 ; 0 ; 0]$ ) 

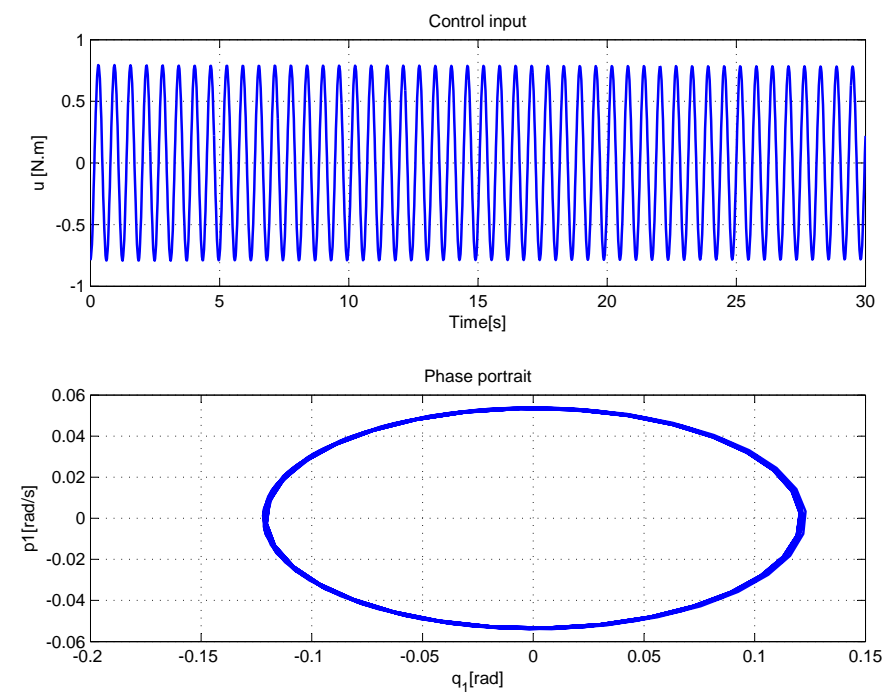

Figure 10. Phase portrait when $k_{1}=k_{1,1}^{c}$

\subsubsection{Case 2 : $k_{1}=k_{1,2}^{c}$}

Similarly uncertainties and external disturbances were neglected in this case. The proposed simulation started from the initial condition given in Table 5. Then, application of the control law (10) to the nominal nonlinear dynamics of the IWIP gave results depicted in figures (11) and (12). The former displays the pendulum joint position and velocity versus time. These results clearly show the convergence of the trajectory of the controlled IWIP to the desired stable period-1 limit cycle. It can be observed from the displayed results that the period of the reference limit cycle is about $0.2388[s]$. The latter represents the control law and the phase portrait showing clearly the convergence from the initial condition to a stable limit cycle.
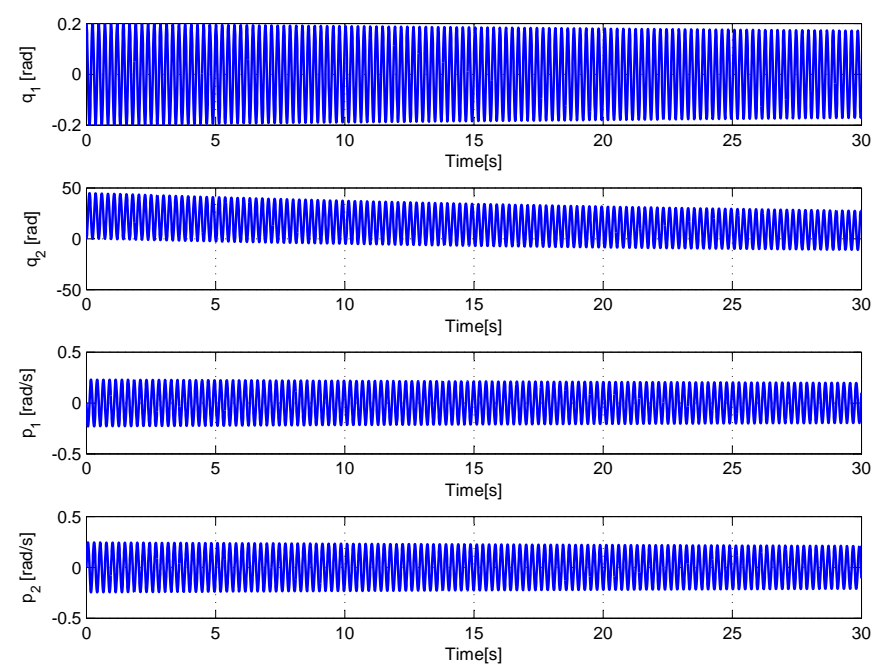

Figure 11. Pendulum joint position and velocity versus time 

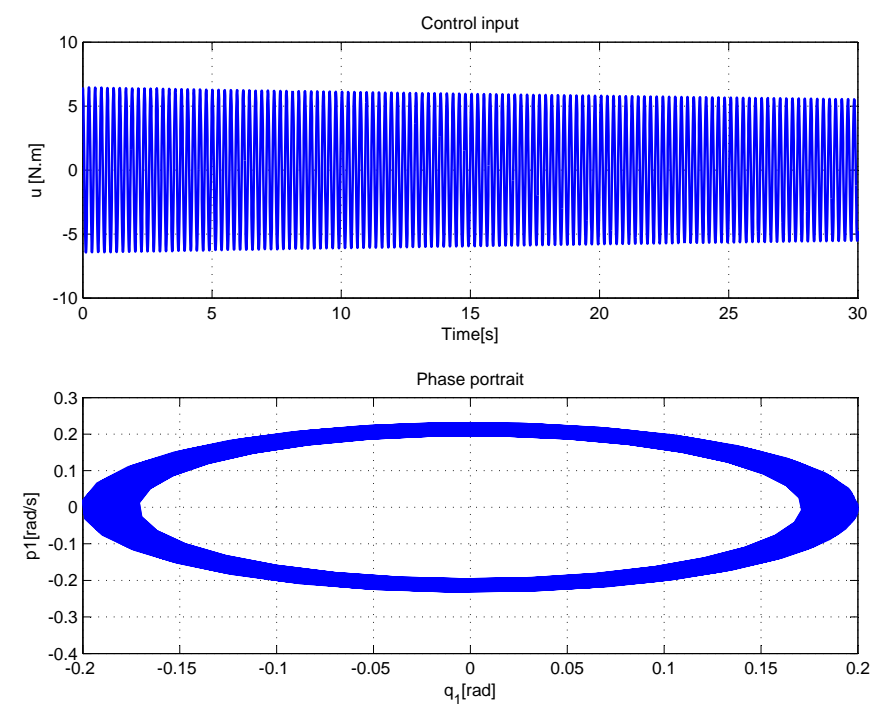

Figure 12. Phase portrait when $k_{1}=k_{1,2}^{c}$

\section{Conclusion and future works}

In this work we studied the dynamical behaviour of a conventional inertia wheel inverted pendulum with a disk in its free extreme. The system is underactuated since the pendulum can rotate freely around its pivot point. It was controlled by the well known IDA-PBC controller which stabilize the system at its upward position (unstable equilibrium). The designed control law was based on some control parameters such as a proportional gain and a damping injection gain. The main investigation of this paper deals with the following question: Is the dynamic properties (stabilization) of the C-IWIP change when varying the control gains $k_{1}, k_{2}, k_{3}$, and $k_{4}$ ?

Our main contribution consists of the use of the Hopf bifurcation theory and the center manifold theorem to show some additional dynamical behaviours of the inverted pendulum. We analyzed stability of the equilibrium point of the C-IWIP and then two stability conditions were developed. In addition, we determined the existence condition of the Hopf bifurcation as a function of some gain parameters of the IDA-PBC and therefore the generation of periodic oscillations (limit cycles). We characterized the stability of such solutions analytically by knowing the sign of the first Lyapunov value at the bifurcation boundary.Furthermore, we proved the effectiveness of analytical results numerically (Hopf bifurcation detection and stable limit cycle location).

In this work, analytical and numerical results were developed when applying IDA-PBC to the IWIP. However, to look for stable limit cycles the same methodology can be easily used to other underactuated mechanical systems with the same controller or with other controllers. As a future direction, we look for designing a robust tracking controller for the IWIP with uncertain parameters and/or under external disturbances. In addition, bifurcation analysis based on hamiltonian systems can be investigated.

\section{References}

Alonso, D. M., Paolini, E. E. \& Moiola, J. L. [2002] Controlling an inverted pendulum with bounded controls (Lecture Notes in Control and Information Sciences, Springer-Verlag).

Alonso, D. M., Paolini, E. E. \& Moiola, J. L. [2005] "Global bifurcation analysis of a controlled underactuated mechanical system," Nonlinear Dynamics 40, 205-225.

Andary, S., Chemori, A., Benoit, M. \& Sallantin, J. [2012] "A dual model-free control of underactuated mechanical systems, application to the inertia wheel inverted pendulum," Proc. Proceedings of American Control Conference (ACC'12) (Fairmont Queen Elizabeth, Montréal, Canada).

Andary, S., Chemori, A. \& Krut, S. [2009a] "Control of the underactuated inertia wheel inverted pendulum for stable limit cycle generation," RSJ Advanced Robotics 23, 1999-2014. 
Andary, S., Chemori, A. \& Krut, S. [2009b] "Estimation-based disturbance rejection in control for limit cycle generation on inertia wheel inverted pendulum testbed," Proc. Proceedings of the 2009 IEEE/RSJ international conference on Intelligent robots and systems(IROS'09) (Piscataway, NJ, USA), pp. 13021307.

Choukchou-Braham, A., Cherki, B., Djemaï, M. \& Busawon, K. [2014] Analysis and Control of Underactuated Mechanical Systems (Springer-Verlag, Switzerland).

Dhooge, A., Govaerts, W. \& Kuznetsov, Y. A. [2003] "Matcont: A matlab package for numerical bifurcation analysis of odes," ACM Transactions on Mathematical Software 29(2), 141-164.

Fradkov, A. \& Pogromsky, A. [1998] "Introduction to control of oscillations and chaos," World Scientific .

Guckenheimer, J. \& Holmes, P. [1983] Nonlinear oscillations, dynamical systems, and bifurcations of vector fields (Springer-Verlag, New York).

Khraief-Haddad, N., Chemori, A. \& Belghith, S. [2014] "External disturbance rejection in ida-pbc controller for underactuated mechanical systems: From theory to real-time experiments," IEEE MultiConference on Systems and Control - MSC'14 (Antibes, France).

Khraief-Haddad, N., Chemori, A., Pena, J. J. \& S.Belghith [2015] "Stabilization of inertia wheel inverted pendulum by model reference adaptive ida-pbc : From simulation to real-time experiments," 3rd International Conference on Control, Engineering and Information Technology - CEIT'15 (Tlemcen, Algeria).

Kuehn, C. [2010] "From first Lyapunov coefficients to maximal canards," International Journal of Bifurcation and Chaos 20((5), 1467-1475.

Kuznetsov, Y. [1998] Elements of Applied Bifurcation Theory (Springer-Verlag, New York).

Leonard, N., Bloch, A. \& Marsden, J. [1998] Mechanical Feedback Control Systems.In M. Vidyasagar V.D. Blondel, E.D. Sontag and J.C. Willems, editors, Open Problems in Mathe-matical Systems and Control Theory (Springer-Verlag, New York).

Marsden, J. \& McCracken, M. [1976] The Hopf Bifurcation and Its Applications (Springer-Verlag).

Nikolov, S. \& Nedev, V. [2016] "Bifurcation analysis and dynamic behaviour of an inverted pendulum with bounded control," Journal of Theoretical and Applied Mechanics 64(1), 17-32.

Ortega, R., Spong, M., Gomez-Estern, F. \& Blankenstein, G. [2002] "Stabilization of a class of underactuated mechanical systems via interconnection and damping assignment," IEEE Transactions on Automatic Control 48, 1218-1233.

Pagano, D., Pizarro, L. \& Aracil, J. [2000] "Local bifurcation analysis in the furuta pendulum via normal forms," International Journal of Bifurcation and Chaos 10(5), 981-995.

Palacios, A. F. [2006] The Hamilton-Type Principle in Fluid Dynamics (Springer-Verlag, New York).

Santibañez, V., Kelly, R. \& Sandoval, J. [2005] "Control of the inertia wheel pendulum by bounded torques," Decision and Control, 2005 and 2005 European Control Conference. CDC-ECC'05. 44th IEEE Conference on (IEEE), pp. 8266-8270.

Spong, M. \& Vidyasagar, M. [1989] Robot Dynamics and Control (John Wiley and Sons, New York, USA).

Wiggins, S. [2003] Introduction to Applied Nonlinear Dynamical systems and Chaos (Springer-Verlag, New York). 